\title{
Solving Nonlinear Heat Transfer Problems Using Variation of Parameters
}

Travis J. Moore

Brigham Young University - Provo, travisjmoore@gmail.com

Matthew R. Jones

Brigham Young University - Provo, mrjones@byu.edu

Follow this and additional works at: https://scholarsarchive.byu.edu/facpub

Part of the Engineering Commons

\section{Original Publication Citation}

Travis J. Moore, Matthew R. Jones, Solving nonlinear heat transfer problems using variation of parameters, International Journal of Thermal Sciences, Volume 93, 2015, Pages 29-35, ISSN 1290-0729, https://doi.org/10.1016/j.ijthermalsci.2015.02.002. (http://www.sciencedirect.com/ science/article/pii/S1290072915000538)

\section{BYU ScholarsArchive Citation}

Moore, Travis J. and Jones, Matthew R., "Solving Nonlinear Heat Transfer Problems Using Variation of Parameters" (2015). Faculty Publications. 3155.

https://scholarsarchive.byu.edu/facpub/3155

This Peer-Reviewed Article is brought to you for free and open access by BYU ScholarsArchive. It has been accepted for inclusion in Faculty Publications by an authorized administrator of BYU ScholarsArchive. For more information, please contact ellen_amatangelo@byu.edu. 


\section{Solving nonlinear heat transfer problems using variation of Q3 parameters}

Q2 Travis J. Moore*, Matthew R. Jones

Department of Mechanical Engineering, Brigham Young University, Provo, UT, USA

\section{A R T I C L E I N F O}

\section{Article history:}

Received 23 August 2014

Received in revised form

6 February 2015

Accepted 9 February 2015

\section{Keywords:}

Variation of parameters

Nonlinear differential equations

Heat transfer applications
Available online $\mathrm{xxx}$

\begin{abstract}
A B S T R A C T
Nonlinear problems arise in many heat transfer applications, and several analytical and numerical methods for solving these problems are described in the literature. Here, the method of variation of parameters is shown to be a relatively simple method for obtaining solutions to four specific heat transfer problems: 1 . a radiating annular fin, 2. conduction-radiation in a plane-parallel medium, 3. convective and radiative exchange between the surface of a continuously moving strip and its surroundings, and 4 . convection from a fin with temperature-dependent thermal conductivity and variable cross-sectional area. The results for each of these examples are compared to those obtained using other analytical and numerical methods. The accuracy of the method is limited only by the accuracy with which the numerical integration is performed. The method of variation of parameters is less complex and relatively easy to implement compared to other analytical methods and some numerical methods. It is slightly more computationally expensive than traditional numerical approaches. The method presented may be used to verify numerical solutions to nonlinear heat transfer problems.
\end{abstract}

(c) 2015 Published by Elsevier Masson SAS.

\section{Introduction}

Modeling of heat transfer processes often results in the development of nonlinear differential equations. Specifically, application of an energy balance to a system frequently results in a nonlinear differential equation that governs the temperature field in the system. Phenomena that give rise to nonlinear differential equations include radiative exchange between surfaces, temperaturedependent properties, modeling the dependence of a convective heat transfer coefficient on temperature, and the coupling of the participating media. In general, nonlinear differential equations do not have analytical, closed-form solutions, so they are typically solved using numerical methods. Best practice requires verification of numerical solutions, and the analytical approach described in this paper is a tool for verifying algorithms used to obtain numerical solutions of nonlinear heat transfer problems.

Other analytical methods have been used to solve the nonlinear differential equations that arise in heat transfer applications. One of the most common nonlinear heat transfer problems comes from

Q1 * Corresponding author.

E-mail address: travisjmoore@gmail.com (T.J. Moore). energy equation with the total radiative heat flux in radiatively the analysis of extended surfaces. Abbasbandy and Shivanian [1] obtained an exact analytical solution to the convective fin problem in which the local convection coefficient along the fin surface has a power-law-type dependence on the local temperature difference between the fin and the surrounding fluid. Arslanturk [2] used the Adomian decomposition method (ADM) to analyze a convective fin with temperature-dependent thermal conductivity while Moradi and Ahmadikia [3] solved a similar problem for fins of variable cross-sectional area using the differential transformation method (DTM). Aziz and Khani [4] used the homotopy analysis method (HAM) to solve the nonlinear equation describing the temperature distribution in a continuously moving radiativeconvective fin with temperature-dependent thermal conductivity. The temperature distribution in convective annular fins with temperature-dependent thermal conductivity has been found by Ganji et al. [5] using the homotopy perturbation method (HPM) while that of radiating annular fins has been found using a Green's function approach [6-8]. The advantages of many of these analytical approaches over numerical methods are their direct applicability to both linear and nonlinear equations without requiring linearization, discretization, or perturbation [3]. However, implementation of these complex methods requires the use of infinite power series. 
In addition to extended surfaces, nonlinear differential equations arise when analyzing the energy equation in a radiatively participating medium. These problems are highly nonlinear because the energy equation requires the total radiative heat flux, which is found by solving the integro-differential radiative transfer equation (RTE) [9]. These problems are encountered in the analysis of combustion chambers, rocket nozzles, high-temperature heat exchangers, translucent glass or ceramic coatings, porous insulation, heat treatment of glass plates, and the drawing of optical fibers. The combined conduction-radiation problem has been solved using an integral transformation method $[10,11]$, the finite element method [12,13], the finite difference method [14,15], the finite volume method [16,17], the lattice Boltzmann method [18-20], and finite strip theory [21].

The variation of parameters method has been primarily used to solve linear, nonhomogeneous differential equations, but application of this method to solve nonlinear differential equations has been described previously [22-25]. These publications focus on general mathematical aspects of the solution, particularly application of the method to problems involving an inhomogeneity that is a function of the dependent variable. The contribution of this work is a demonstration that the method of variation of parameters is a relatively simple method of obtaining exact solutions to the nonlinear differential equations that arise in a variety of heat transfer applications. In addition to its intrinsic value as an analytical solution procedure, this approach may be used to verify solutions obtained using more computationally efficient numerical methods.

Following a brief overview of how the method of variation of parameters may be used to obtain exact solutions of nonlinear equations, application of the method is illustrated by solving models derived from analysis of four heat transfer applications. Application of the method to the models developed for the first three problems results in an integral equation that is solved using numerical quadrature. Although numerical methods are used, it should be noted that numerical integration may be performed to an arbitrary degree of precision, so while not closed-form, these solutions can be considered exact. The last example requires finite difference approximations of derivatives, so it is not an exact solution. These solutions are compared with solutions obtained using other analytical or numerical methods. These examples demonstrate that variation of parameters is an easily implemented method of solving the nonlinear differential equations that result from the analysis of a wide range of heat transfer applications.

\section{Method of variation of parameters}

Consider the following second order partial differential equation

$\frac{d^{2} y}{d x^{2}}+C \frac{d y}{d x}+D y=f(x, y), \quad x_{1} \leq x \leq x_{2}$

where $C$ and $D$ are constants and $f$ is a nonlinear function of the independent variable $x$ and the dependent variable $y$. The solution to the boundary value problem in Equation (1) consists of the sum of the complementary and particular solutions. The complementary solution is the solution to the homogeneous equation corresponding to Equation (1) that may be found using traditional solution techniques [26]. The complementary solution is

$y_{c}(x)=c_{1} y_{1}(x)+c_{2} y_{2}(x)$ where $c_{1}$ and $c_{2}$ are constants and $y_{1}$ and $y_{2}$ form a fundamental set of solutions of the homogeneous equation. The particular solution [26] is

$y_{p}=u_{1}(x, y) y_{1}(x)+u_{2}(x, y) y_{2}(x)$

where

$u_{1}(x, y)=-\int_{x_{1}}^{x} \frac{f(t, y) y_{2}(t)}{W\left(y_{1}, y_{2}\right)} d t$

$u_{2}(x, y)=\int_{x_{1}}^{x} \frac{f(t, y) y_{1}(t)}{W\left(y_{1}, y_{2}\right)} d t$

$W\left(y_{1}, y_{2}\right)=\left|\begin{array}{ll}y_{1} & y_{2} \\ y_{1}^{\prime} & y_{2}^{\prime}\end{array}\right|=y_{1} y_{2}^{\prime}-y_{2} y_{1}^{\prime}$

The solution to Equation (1) is therefore

$y(x)=c_{1} y_{1}(x)+c_{2} y_{2}(x)+u_{1}(x, y) y_{1}(x)+u_{2}(x, y) y_{2}(x)$

The constants $c_{1}$ and $c_{2}$ are found from the boundary conditions. Because $u_{1}$ and $u_{2}$ are functions of the dependent variable, $y$, an iterative approach using the method of successive approximations is required to determine $y$, and numerical integration of Equations (4) and (5) is required. Note that $f$ may be a function of the derivatives of $y$. In such cases, the derivatives of $y$ are approximated using finite difference equations.

In order to assess the accuracy and computational efficiency of this approach relative to the accuracy and computational efficiency of numerical methods, the variation of parameters solution and the finite difference solution of Equation (8) are compared to its exact solution [27].

$y^{\prime \prime}=x+y$

Note that in this equation, the inhomogeneity is a function of both the dependent and independent variables. If the $y$ on the right side is moved to the left side, this equation becomes a simple ordinary, linear, nonhomogeneous differential equations which may be solved exactly. Solutions were obtained for various boundary conditions, the average error in the variation of parameters solution ranged from $2.6 \%$ when 10 steps were used in the numerical quadrature to $0.0007 \%$ when 1000 steps were used in the numerical quadrature. Clearly, the accuracy of the variation of parameters approach is limited only by the accuracy of the numerical integration. Complete details regarding the comparisons between the exact solution and the variation of parameters solution are available in Ref. [27].

This problem was also solved using a finite difference method. For a given step size, the average error between the exact solution and that found using finite difference methods was the same as the error between the exact solution and the variation of parameters solution to four significant figures. The complexity of the implementation of the method of variation of parameters was comparable to that of the finite difference approach and the computational time required by the method of variation of parameters method was slightly larger than that required by the finite difference approach.

\section{Example 1. Radiating Annular Fin}

Heat flux measurements are important in many processes and procedures. These include monitoring ovens, furnaces, and kilns, flammability testing, measuring the thermal properties of 
materials, assessing heating, ventilation, and air conditioning systems, and validation of numerical models of combustion processes. Circular foil heat flux gauges can be modeled as radiating annular fins. Liechty et al. [8] presented a thermal model of a copperconstantan circular foil heat flux gauge exposed to a blackbody source in a vacuum environment. Such a setup is typically used to calibrate a heat flux gauge. The nondimensional governing equation for the dimensionless temperature profile and the boundary conditions for this model are given as follows.

$\frac{1}{\rho} \frac{d}{d \rho}\left(\rho \frac{d \theta}{d \rho}\right)=A \theta^{4}-A B$

$\theta(1)=1$

$\left.\frac{d \theta}{d \rho}\right|_{\rho=0}=0$

In Equation (9), $\theta$ is the dimensionless temperature, $\rho$ is the dimensionless radial coordinate, $A$ is the dimensionless gauge parameter, and $B$ is the dimensionless irradiation. The solution to the homogeneous equation corresponding to Equation (9) is found by separating and integrating twice.

$\theta_{c}=c_{1} \ln \rho+c_{2}$

The particular solution is found by using the method of variation of parameters and is given by

$\theta_{p}(\rho)=\ln \rho \int_{0}^{\rho} t\left(A \theta^{4}-A B\right) d t-\int_{0}^{\rho} t\left(A \theta^{4}-A B\right)(\ln t) d t$

The dimensionless temperature profile is the sum of the complementary and particular solutions. Applying the boundary conditions provides expressions for the constants and the dimensionless temperature profile is given as follows.

$$
\begin{aligned}
\theta(\rho)= & +\int_{0}^{1} t\left(A \theta^{4}-A B\right)(\ln t) d t+\ln \rho \int_{0}^{\rho} t\left(A \theta^{4}-A B\right) d t \\
& -\int_{0}^{\rho} t\left(A \theta^{4}-A B\right)(\ln t) d t
\end{aligned}
$$

Equation (14) was solved iteratively using the method of successive approximations. Fig. 1 shows a comparison of the dimensionless temperature profiles calculated from Equation (14) to those found using the Green's function method used by Liechty et al. [8] and to those found from a direct numerical solution using commercial software [28] for various values of the parameters $A$ and $B$. The accuracy and computational requirements of the method of variation of parameters were compared to those of finite difference methods. Because of the highly nonlinear nature of the governing equation, iterative approaches such as Newton's method or the relaxation method must be used with the finite difference equations. This increases the computational expense and decreases the accuracy of the finite difference method. It also made the implementation of this method more complicated than the method of variation of parameters. Because no closed-form solution is possible for this equation, the accuracy cannot be determined. However, for a given step size, the results of both approaches were the same to three significant figures. Again, the computational time

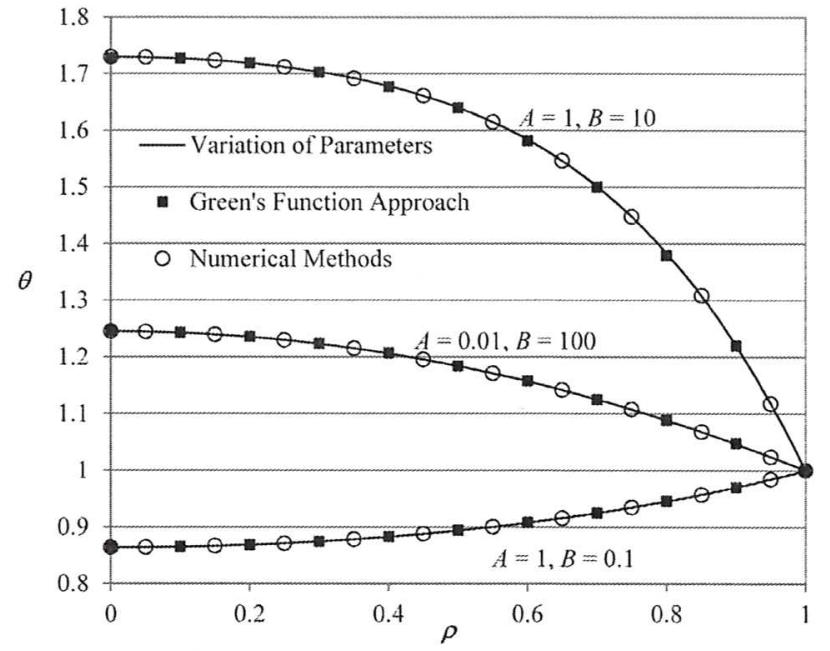

Fig. 1. Comparison of the dimensionless temperature profiles found from Equation (14) to those found from a Green's function approach and numerical methods for various values of the parameters $A$ and $B$.

required was slightly higher for the method of variation of parameters.

Example 2. Combined Conduction-Radiation Problem

A radiatively participating plane-parallel medium in which conduction is significant can be used to model translucent coatings. Examples of the application of such coatings include thermal barrier coatings (TBCs) which are used in power generation and aerospace turbines to protect super alloy components from large and extended heat loads, and glass coatings that are used as sacrificial ablating coatings to protect the interior of a body from high external temperatures. The method of variation of parameters has been used to analyze the conduction-radiation problem in a non-scattering, non-gray medium with non-gray opaque boundaries [29]. For simplicity in demonstrating this method here, the simplest case of a gray medium with black boundaries, as shown in

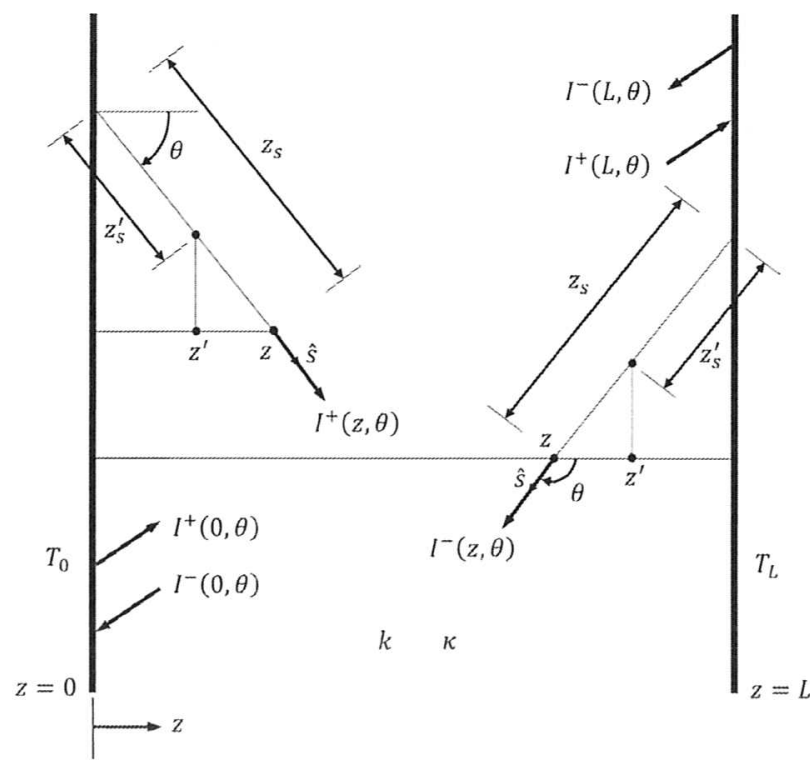

Fig. 2. Coordinate system used in the analysis of a gray medium between black boundaries. 
Fig. 2, is analyzed. For a one-dimensional, planar medium at steady state and without internal heat generation, the energy equation reduces to [9].

$\frac{d^{2} T}{d z^{2}}=\frac{1}{k} \frac{d q_{R}}{d z}$

The surrounding black surfaces are at specified temperatures $T(0)=T_{0}$ and $T(L)=T_{L}$. The complementary solution to Equation (15) is found by separating and integrating the corresponding homogeneous equation twice, which yields

$T_{c}=c_{1} z+c_{2}$

The particular solution requires determination of the total radiative heat flux, $q_{R}$. Assuming azimuthal symmetry and applying the variable transformation $\mu=\cos \theta$, the total radiative heat flux may be expressed as [9].

$q_{R}=2 \pi \int_{-1}^{+1} I(z, \mu) \mu d \mu=2 \pi\left(\int_{0}^{1} I^{+}(z, \mu) \mu d \mu-\int_{0}^{1} I^{-}(z,-\mu) \mu d \mu\right)$

The radiative transfer equation (RTE) is used to calculate $I^{+}(z, \mu)$ and $I^{-}(z,-\mu)$. The details of these solutions can be found in [9] and [29].

$I^{+}(z, \mu)=I_{b}(T(0)) e^{-\kappa z / \mu}+\frac{\kappa}{\mu} \int_{0}^{z} I_{b}(T(t)) e^{-\kappa(z-t) / \mu} d t$

$I^{-}(z,-\mu)=I_{b}(T(L)) e^{-\kappa(L-z) / \mu}+\frac{\kappa}{\mu} \int_{z}^{L} I_{b}(T(t)) e^{-\kappa(t-z) / \mu} d t$

Equations (18) and (19) are substituted into Equation (17) which is, in turn, substituted into Equation (15). The method of variation of parameters is used to find the particular solution to Equation (15). The details of this solution are omitted here for brevity, but are provided in [29]. An expression for the temperature profile is found by summing the complementary and particular solutions and applying the boundary conditions and is shown in Equation (20).

$T(z)=\left(\frac{T_{L}}{L}-\frac{T_{0}}{L}\right) z+T_{0}+\frac{2}{k} \sum_{i=1}^{6} \int_{0}^{1} H_{i}(z, \mu) d \mu$

The terms in the summation in Equation (20) are shown in Table 1, where

$P(z, \mu)=\frac{Z}{L}\left(e^{-\kappa L / \mu}-1\right)+\left(1-e^{-\kappa z / \mu}\right)$

$Q(z, \mu)=\frac{Z}{L}\left(1-e^{-\kappa L / \mu}\right)+e^{-\kappa L / \mu}\left(1-e^{\kappa z / \mu}\right)$

The accuracy of the method is verified by comparing the results for various cases to those calculated using different numerical

Table 1

Terms in the summation of Equation (20).

\begin{tabular}{ll}
\hline$H_{1}=1 / \kappa E_{b}(T(0)) P(z, \mu) \mu^{2}$ & $H_{4}=1 / \kappa E_{b}(T(L)) Q(z, \mu) \mu^{2}$ \\
$H_{2}=z e^{-\kappa L / \mu} \mu \int_{0}^{L} E_{b}(T(t)) e^{\kappa t / \mu} d t$ & $H_{5}=\left(1-\frac{z}{L}\right) \int_{0}^{L} E_{b}(T(t)) e^{-\kappa t / \mu} \mu d t$ \\
$H_{3}=-e^{-\kappa z / \mu} \mu \int_{0}^{z} E_{b}(T(t)) e^{\kappa t / \mu} d t$ & $H_{6}=-e^{\kappa z / \mu} \mu \int_{z}^{L} E_{b}(T(t)) e^{-\kappa t / \mu} d t$ \\
\hline
\end{tabular}

methods, including CFD simulations in which the discreteordinates method is used to model the radiative heat transfer [30]. The conduction-to-radiation parameter is defined as

$N=\frac{k \kappa}{4 \sigma T_{0}^{3}}$

This parameter provides a good estimate for the relative importance of conduction heat transfer relative to radiation heat transfer [9]. For the case where $T_{L}=0.5 T_{0}$ and $K=1$, the nondimensional temperature profiles calculated using Equation (20) are compared to those calculated using a finite difference method [8], the collapsed dimension method implemented by Talukdar and Mishra [31], and the discrete ordinates method [30]. Fig. 3 shows these comparisons for different values of $N$. A discussion of the comparison of the computational expense for these different methods is available in [29].

Example 3. Convective and Radiative Exchange between the Surface of a Continuously Moving Strip and its Surroundings

In processes such as extrusion, hot rolling, and casting, the manufactured material loses heat to its surroundings by convection and radiation while in continuous motion. This heat transfer process is designed to cool the material to a specified temperature before it is spooled or removed. Aziz and Khani [4] used the homotopy analysis method (HAM) to find an analytic solution for heat transfer in a continuously moving strip which is losing heat to its surrounding by simultaneous convection and radiation. The nondimensional governing equation and boundary conditions to this problem are given as follows.

$\frac{d^{2} \theta}{d X^{2}}-N_{c}\left(\theta-\theta_{a}\right)-N_{r}\left(\theta^{4}-\theta_{a}^{4}\right)-P e \frac{d \theta}{d X}=0$

$\theta(0)=1$

$\left.\frac{d \theta}{d X}\right|_{X=L^{*}}=0$

In these equations, $\theta$ is the dimensionless temperature, $X$ is the dimensionless position along the strip, $N_{c}$ is the convectionconduction number (commonly known as the Biot number), $N_{r}$ is the radiation-conduction number, $P e$ is the Peclet number (which represents the dimensionless speed of the moving strip), $L^{*}$ is a

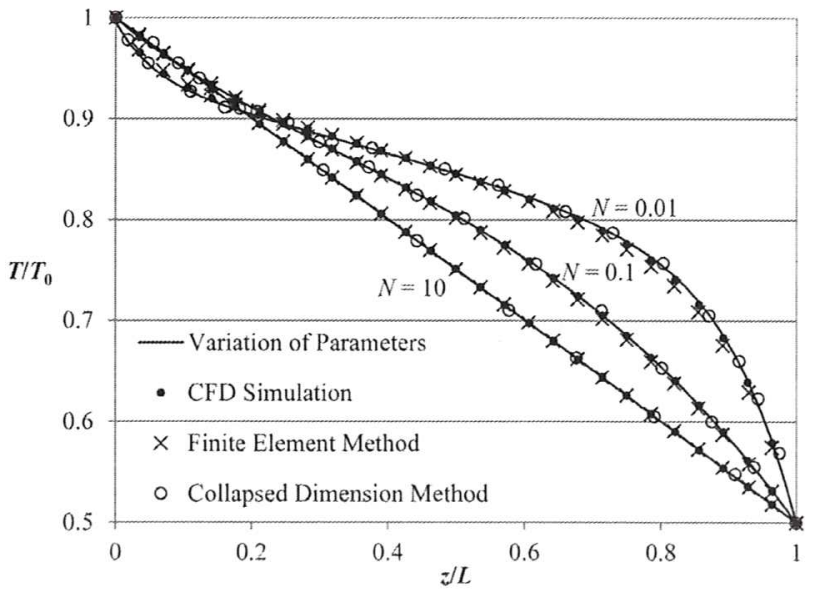

Fig. 3. Comparison of the variation of parameters method to numerical methods for a gray medium $(K=1)$ with black boundaries and $T_{L}=0.5 T_{0}$ for various conduction-toradiation parameters. 
parameter that allows the length of the strip that is exposed to the surrounding fluid to be adjusted, and the subscript $a$ refers to the temperature of the surroundings. Rearranging Equation (24) to match the form of Equation (1) gives

$\frac{d^{2} \theta}{d X^{2}}-P e \frac{d \theta}{d X}-N_{c} \theta=N_{r}\left(\theta^{4}-\theta_{a}^{4}\right)-N_{c} \theta_{a}$

The solution to the homogeneous equation corresponding to Equation (27) may be obtained using an auxiliary equation [25].

$\theta_{C}=c_{1} e^{m_{1} X}+c_{2} e^{m_{2} X}$

where the roots of the auxiliary equation are given by

$m_{1}=\frac{P e+\sqrt{(P e)^{2}+4 N_{c}}}{2}$

$m_{2}=\frac{P e-\sqrt{(P e)^{2}+4 N_{c}}}{2}$

Variation of parameters is used to find the particular solution, which is

$$
\begin{aligned}
\theta_{p}(X)= & -e^{m_{1} X} \int_{0}^{X} \frac{\left(N_{r}\left(\theta^{4}-\theta_{a}^{4}\right)-N_{c} \theta_{a}\right) e^{m_{2} t}}{\left(m_{2}-m_{1}\right) e^{\left(m_{1}+m_{2}\right) t}} d t \\
& +e^{m_{2} X} \int_{0}^{X} \frac{\left(N_{r}\left(\theta^{4}-\theta_{a}^{4}\right)-N_{c} \theta_{a}\right) e^{m_{1} t}}{\left(m_{2}-m_{1}\right) e^{\left(m_{1}+m_{2}\right) t}} d t
\end{aligned}
$$

The dimensionless temperature profile is the sum of the complementary and particular solutions. Applying the boundary conditions provides expressions for the constants and the dimensionless temperature profile is

$$
\begin{aligned}
\theta(X)= & c_{1} e^{m_{1} X}+c_{2} e^{m_{2} X}-e^{m_{1} X} \int_{0}^{X} \frac{\left(N_{r}\left(\theta^{4}-\theta_{a}^{4}\right)-N_{c} \theta_{a}\right) e^{m_{2} t}}{\left(m_{2}-m_{1}\right) e^{\left(m_{1}+m_{2}\right) t}} d t \\
& +e^{m_{2} X} \int_{0}^{X} \frac{\left(N_{r}\left(\theta^{4}-\theta_{a}^{4}\right)-N_{c} \theta_{a}\right) e^{m_{1} t}}{\left(m_{2}-m_{1}\right) e^{\left(m_{1}+m_{2}\right) t}} d t
\end{aligned}
$$

where slightly higher. The solutions were the same to three significant figures.

Example 4. Exponential Convective Fin with TemperatureDependent Thermal Conductivity

Heat transfer through extended surfaces is used in many industrial applications. An extensive review of this subject is given by Kraus et al. [32]. The use of fins with constant cross-sectional area and the assumption of constant thermophysical properties over the length of the fin allow for closed-form analytical solutions to the energy equation [32]. However, fins optimized for specific purposes often have variable cross-sectional area and, if a large temperature difference exists within the fin, the thermal conductivity of the fin may vary over the length of the fin. These complexities significantly complicate the heat transfer model, resulting in a nonlinear differential equation. Moradi and Ahmadikia [3] used the differential transformation method (DTM) to find an analytical solution to the energy equation for fins with varying cross sectional areas and with temperature-dependent thermal conductivity. Here, variation of parameters is used to calculate the temperature profile in a fin with an exponential profile and a thermal conductivity that varies linearly with temperature. The nondimensional governing equation and boundary conditions are given by [3].

$\frac{d}{d X}\left(e^{a X}(1+\beta \theta) \frac{d \theta}{d X}\right)-N^{2} \theta=0$

$\theta(1)=1$

$\left.\frac{d \theta}{d X}\right|_{X=0}=0$

In the above equations, $\theta$ is the dimensionless temperature, $\mathrm{X}$ is the dimensionless position, $\mathrm{N}$ is the dimensionless fin parameter, which is a measure of the ratio of convection to conduction, a governs the shape of the exponential profile of the fin, and $\beta$ governs the relationship between the fin temperature and the thermal conductivity. Equation (35) can be expanded and rearranged such that it is in the form of Equation (1).

$\frac{d^{2} \theta}{d X^{2}}=\frac{1}{e^{a X}(1+\beta \theta)}\left(N^{2} \theta-e^{a X} \beta\left(\frac{d \theta}{d X}\right)^{2}\right)-a \frac{d \theta}{d X}$

The derivatives in the nonhomogeneity must be approximated using finite difference methods. Because the derivatives are $c_{2}=1-c_{1}$

Fig. 4 shows a comparison of the dimensionless temperature profiles calculated from Equation (32) to those found using HAM used by Aziz and Khani [4] and to those found from a direct numerical solution using commercial software [28] for $\mathrm{N}_{\mathrm{c}}=4, \mathrm{~N}_{\mathrm{r}}=4$, $\mathrm{Pe}=3$, and $\mathrm{L}^{*}=1$. The problem was also solved using finite difference methods. Again, the complexity of implementing the method of variation of parameters was comparable to that of the finite difference method, and the computational expense was approximate, this solution cannot be considered exact, unlike the first three examples. The homogeneous equation corresponding to Equation (38) is separated and integrated twice, yielding the complementary solution.

$\theta_{c}=c_{1} X+c_{2}$

The particular solution is found using the method of variation of parameters and is given as 


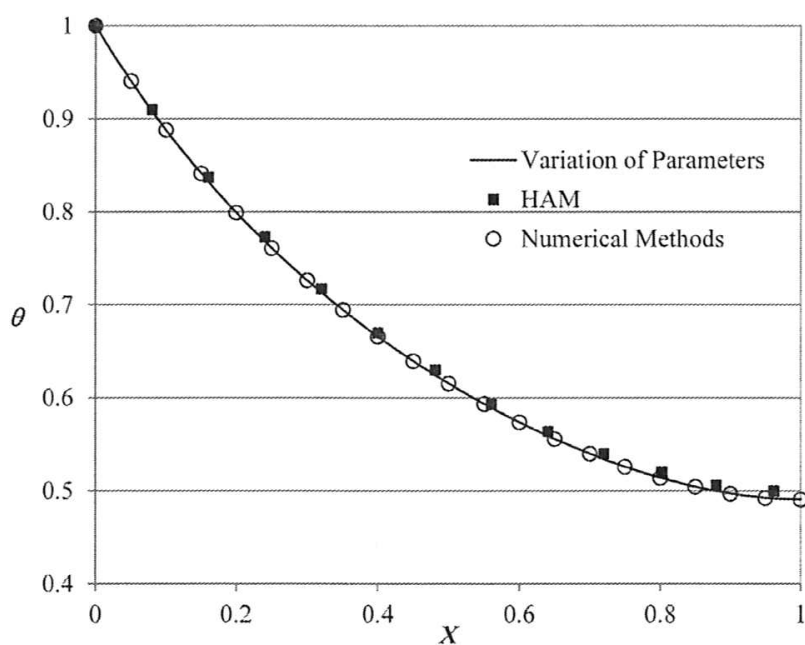

Fig. 4. Comparison of dimensionless temperature profiles found from Equation (32) to those found from HAM and numerical methods for $N_{c}=4, N_{r}=4, P e=3$, and $L^{*}=1$.

$\theta_{p}(X)=X \int_{0}^{X} f\left(t, \theta, \theta^{\prime}\right) d t-\int_{0}^{X} t f\left(t, \theta, \theta^{\prime}\right) d t$

where

$f\left(X, \theta, \theta^{\prime}\right)=\frac{1}{e^{a X}(1+\beta \theta)}\left(N^{2} \theta-e^{a X} \beta\left(\theta^{\prime}\right)^{2}\right)-a \theta^{\prime}$

The dimensionless temperature profile is the sum of the complementary and particular solutions. The constants in Equation (39) are found by applying the boundary conditions so that the nondimensional temperature profile is given as follows.

$$
\begin{aligned}
\theta(X)= & 1-\int_{0}^{1} f\left(t, \theta, \theta^{\prime}\right) d t+\int_{0}^{1} t f\left(t, \theta, \theta^{\prime}\right) d t+X \int_{0}^{X} f\left(t, \theta, \theta^{\prime}\right) d t \\
& -\int_{0}^{X} t f\left(t, \theta, \theta^{\prime}\right) d t
\end{aligned}
$$

Fig. 5 compares the temperature profile calculated from Equation (42) to that calculated using DTM [3] and that found from the direct numerical solution of Equation (35) [28] for various values of $\beta$ with $\mathrm{a}=1$ and $\mathrm{N}=1$. Comparison of the complexity of implementation and accuracy of the method of variation of parameters with that of a finite difference method lead to the same conclusions as were drawn in the previous examples.

\section{Summary and conclusions}

Analysis of a wide variety of heat transfer problems leads to the derivation of nonlinear differential equations, and a range of analytical and numerical methods for solving these problems are described in the literature. An analytical solution to this class of problems that is based on the method of variation of parameters has been presented in this paper. Comparison of an exact solution of a linear differential equation to the solution obtained using the method of variation of parameters indicates that the accuracy of the variation of parameters method is limited only by the accuracy to which the numerical quadrature is performed. Since, in principle,

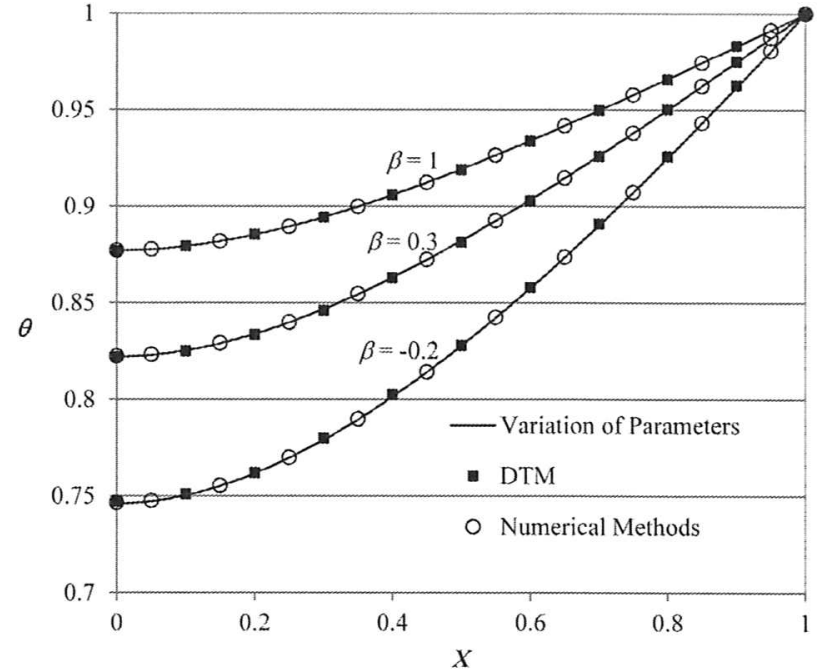

Fig. 5. Comparison of the dimensionless temperature profiles found from Equation (42) to those found from DTM and numerical methods for $a=1$ and $N=1$ for various values of $\beta$.

numerical quadrature may be performed to any accuracy, the variation of parameters method may be considered exact.

Use of the variation of parameters method has been illustrated by applying it to four heat transfer problems. The results of these examples demonstrate that the variation of parameters method is a relatively simple method for obtaining exact solutions to nonlinear differential equations developed in the analysis of heat transfer systems. In addition to its value as an exact solution method, this approach may be used to verify solutions obtained using more computationally efficient numerical methods.

Solutions obtained for the differential equations arising from analysis of a radiating annular fin, analysis of combined conduction and radiation in a plane-parallel medium, and analysis of convective and radiative exchange between the surface of a continuously moving strip and its surroundings are exact. For three of these applications, the accuracy and the computational expense of the method of variation of parameters were compared with those of a finite difference method. The solutions obtained using the variation of parameters approach agree to three significant figures with finite difference solutions, so both methods have the same accuracy. For the examples considered in this paper, the computational efficiency of the finite difference approach was slightly higher than that of the variation of parameters method. The complexity of implementing both methods was comparable for most of the applications, although obtaining a finite difference solution for highly nonlinear equations required the use of iterative techniques. This observation indicates that implementing the variation of parameters method may be less complex when the problem is highly nonlinear. Implementation of the variation of parameters approach was less complex than analytical methods such as the differential transformation method and the homotopy analysis method.

\section{References}

[1] S. Abbasbandy, E. Shivanian, Exact analytical solution of a nonlinear equation arising in heat transfer, Phys. Lett. A 374 (4) (2010) 567-574.

[2] C. Arslanturk, A decomposition method for fin efficiency of convective straight fins with temperature-dependent thermal conductivity, Int. Commun. Heat Mass Transf. 32 (6) (2005) 831-841.

[3] A. Moradi, H. Ahmadikia, Analytical solution for different profiles of fin with temperature dependent thermal conductivity, Math. Problems Eng. 2010 (2010) 15. Article ID 568263.

[4] A. Aziz, H. Khani, Convection-radiation from a continuously moving fin of variable thermal conductivity, J. Frankl. Inst. 348 (4) (2011) 640-651. 
[5] D.D. Ganji, Z.Z. Ganji, H.D. Ganji, Determination of temperature distribution for annular fins with temperature dependent thermal conductivity by HPM Therm. Sci. 15 (1) (2011) S111-S115.

[6] M.R. Jones, V.P. Solovjov, Green's function approach to nonlinear conduction and surface radiation problems, J. Heat Transf. 132 (2) (2010) 024502 (5 pages).

[7] M.R. Jones, V.P. Solovjov, Green's function approach to nonlinear conduction and surface radiation problems, in: Proceeding of HT2009, 2009 Summer Heat Transfer Conference, HT2009-88033, July 13-19, San Francisco CA, 2009.

[8] B.C. Liechty, M.M. Clark, M.R. Jones, R.S. Larson, B.L. Woolford, Nonlinear thermal model of circular foil heat flux gauges, J. Thermophys. Heat Transf. 21 (3) (2007) 468-474

[9] M.F. Modest, Radiative Heat Transfer, second ed., Academic Press, 2003.

[10] R. Viskanta, R.J. Grosh. Heat transfer by simultaneous conduction and radiation in an absorbing medium, J. Heat Transf. 84 (1) (1962) 63-72.

[11] R. Viskanta, R.J. Grosh, Effect of surface emissivity on heat transfer by simultaneous conduction and radiation, Int. J. Heat Mass Transf. 5 (8) (1962) 729-734.

[12] S.M.H. Sarvari, J.R. Howell, S.H. Mansouri, Inverse boundary design conduction-radiation problem in irregular two-dimensional domains, Numer. Heat. Transf. Part B 44 (3) (2003) 209-224.

[13] L.M. Ruan, M. Xie, H. Qi, W. An, H.P. Tan, Development of a finite element model for coupled radiative and conductive heat transfer in participating media, J. Quantitative Spectrosc. Radiat. Transf. 102 (2) (2006) 190-202.

[14] F. Asllanaj, A. Milandri, G. Jeandel, J.R. Roche. A finite difference solution of non-linear systems of radiative-conductive heat transfer equations, Int. I. Numer. Methods Eng. 54 (11) (2002) 1649-1668.

[15] B. Safavisohi, E. Sharbati, C. Aghanajafi, R.K. Firoozabadi, Finite difference solution for radiative-conductive heat transfer of a semitransparent polycarbonate layer, J. Appl, Polym. Sci. 112 (6) (2009) 3313-3321.

[16] S.V. Patankar, Numerical Heat Transfer and Fluid Flow, first ed., Hemisphere Publishing Corp., 1980.

[17] K. Birgelis, U. Raitums, Convergence of the finite volume method for a conductive-radiative heat transfer problem, Math. Model. Analysis 18 (2) (2013) 274-288.

[18] S.C. Mishra, H.K. Roy, Solving transient conduction-radiation problems using the lattice Boltzmann method and the finite volume method, J. Comput. Phys. 223 (1) (2007) 89-107.
[19] B. Mondal, S.C. Mishra, Application of the lattice Boltzmann method and discrete ordinate method for solving transient conduction and radiation heat transfer problems, Numer. Heat. Transf. A 52 (8) (2007) 757-775.

[20] S.C. Mishra, A. Lankadast, Transient conduction-radiation heat transfer in participating media using the lattice Boltzmann method and the discrete transfer method, Numer. Heat. Transf. A 47 (9) (2005) 935-954.

[21] S.P.C. Marques, E.]. Barbero, J.S.R. Murillo, Analysis of conduction-radiation problem in absorbing and emitting nongray materials, Int. J. Numer Methods Heat Fluid Flow 19 (2) (2009) 165-181.

[22] S.T. Mohyud-Din, M.A. Noor, A. Waheed, Variation of parameters method for initial and boundary value problems, World Appl. Sci. J. 11 (5) (2010) 622-639. International Joumal of Modern Applied Physics 3 (1) (2013) $48-56$.

[23] Rahmatullah, S.T. Mohyud-Din, Variation of parameters method for nonlinear diffusion equations, Int, J. Mod. Appl. Phys. 3 (1) (2013) 48-56.

[24] J. Keckic. Additions to Kamkes treatise VII: variation of parameters for nonlinear second order differential equations, Publikacije Elektrotehničkog fakulteta, Serija Matematika i fizika 544 (1976) 31-36.

[25] M.P. Bogdanova, On a generalization of the method of variation of parameters, Doklady Akademii nauk BSSR 6 (1962) 285-287.

[26] D.G. Zill, M.R. Cullen, Advanced Engineering Mathematics, second ed., Jones and Bartlett Publishers, 2000.

[27] T.J. Moore, Application of Variation of Parameters to Solve Nonlinear Multimode Heat Transfer Problems, PhD dissertation, Brigham Young University, Provo, UT, 2014.

[28] Maple 16.00, O Maplesoft

[29] T.J. Moore, M.R. Jones, Analysis of the conduction-radiation problem in absorbing, emitting, non-gray planar media using an exact method, Int. J. Heat Mass Transf. 73 (2014) 804-809.

[30] ANSYS@ Academic Research, Release 14.5.0.

[31] P. Talukdar, S.C. Mishra, Analysis of conduction-radiation problem in absorbing, emitting and anisotropically scattering media using the collapsed dimension method, Int. J. Heat Mass Transf. 45 (10) (2002) 2159-2168.

[32] A.D. Kraus, A. Aziz, J.R. Welty, Extended Surface Heat Transfer, Wiley, New York, 2002. 